\title{
Controlling Hopf Bifurcation of a New Modified Hyperchaotic Lü System
}

\author{
Ping Cai, ${ }^{1,2}$ Jia-Shi Tang, ${ }^{1}$ and Zhen-Bo Li ${ }^{1}$ \\ ${ }^{1}$ College of Mechanical and Vehicle Engineering, Hunan University, Changsha 410082, China \\ ${ }^{2}$ School of Mathematics and Statistics, Minnan Normal University, Zhangzhou 363000, China \\ Correspondence should be addressed to Ping Cai; caiping0596@163.com
}

Received 23 October 2014; Revised 3 March 2015; Accepted 11 March 2015

Academic Editor: Michael Vynnycky

Copyright (c) 2015 Ping Cai et al. This is an open access article distributed under the Creative Commons Attribution License, which permits unrestricted use, distribution, and reproduction in any medium, provided the original work is properly cited.

Controlling Hopf bifurcation of a new modified hyperchaotic Lü system is investigated in this paper. A hybrid control strategy using both state feedback and parameter control is proposed. The control strategy realizes the delay of Hopf bifurcation. Furthermore, by applying the normal form theory, the stability of the bifurcation is determined. Numerical simulation results are given to support the theoretical analysis.

\section{Introduction}

Chaos and bifurcation are of great importance in many physical, chemical, and biological nonlinear systems. Chaos theory is one of the most significant achievements of nonlinear science. Nowadays complex nonlinear systems are being used in many fields of science and engineering. Most of the recent works have focused on solving complex chaos control, synchronization, and so on [1-4]. Bifurcation control has been a rapidly growing interest by many research works in recent years [5-7]. Hopf bifurcation is a kind of important dynamic bifurcation. Contributions in Hopf bifurcation control mainly focused on amplitude control of limit cycle $[8,9]$, changing the critical points of an existing bifurcation [10], delaying the onset of an inherent bifurcation, and stabilizing an existing bifurcation [11-13], creating a desired bifurcation at a desired location, which is called anticontrol of bifurcation [14-17]. Among these researches, 3D chaotic systems play a leading role, such as Lorenz system [18], Chen system [19], and Lü system [20]. The study of 4D hyperchaotic systems has recently become a hot topic [21-24]. 4D hyperchaotic system has more complicated dynamical behaviors compared to $3 \mathrm{D}$ chaotic system. So the analysis and calculation are also more difficult.
The idea of the work is to design a control law to control Hopf bifurcation in nonlinear system. Consider the following general nonlinear system:

$$
\dot{x}=f(x, \mu), \quad x \in R^{n}, \quad \mu \in R,
$$

where the dot denotes differentiation with respect to time $t$ and $x$ is an $n$-dimensional state vector, while $\mu$ is bifurcation parameter. Let $x_{i}^{*}(i=1,2, \ldots, k)$ be $k$ equilibria of system (1); that is, $f\left(x_{i}^{*}, \mu\right) \equiv 0(i=1,2, \ldots, k)$ for any value of $\mu$. A hybrid control strategy is added to model (1), and then we obtain the following controlled system:

$$
\dot{x}=\alpha f(x, \mu)+(1-\alpha) u(x, \mu), \quad \alpha \in R^{n},
$$

where $\alpha$ is a control parameter and $u$ is state feedback controller. In order for the controlled system (2) to keep all the equilibria unchanged under the control, the following conditions should be satisfied:

$$
u\left(x_{i}^{*}, \mu\right)=0, \quad i=1,2, \ldots, k .
$$


A general formula satisfying condition (3) can be constructed as follows:

$$
\begin{aligned}
& u\left(x, x_{1}^{*}, x_{2}^{*}, \ldots, x_{k}^{*}, \mu\right) \\
& =\prod_{i=1}^{k}\left(x-x_{i}^{*}\right)+\sum_{i=1}^{k}\left(x-x_{i}^{*}\right) \prod_{j=1}^{k}\left(x-x_{j}^{*}\right) \\
& \quad+\sum_{i=1}^{k}\left(x-x_{i}^{*}\right)^{2} \prod_{j=1}^{k}\left(x-x_{j}^{*}\right)+\cdots .
\end{aligned}
$$

Obviously, we use nonlinear feedback with polynomial function in the controller $u$. Generally speaking, linear part of a control strategy is used to shift the bifurcation value, in order to eliminate or delay an existing bifurcation. The nonlinear part, on the other hand, can be designed to change the stability of bifurcation solutions. Controller $u$ involves higher-order terms, which may not be necessary for stability control. It is preferable to have the simplest possible design for engineering applications. In most cases, using fewer components or just one component may be enough to satisfy the predesigned control objectives. So, it is not necessary to take all the components in the controller $u$ for practical system. This greatly simplifies the control formula. For example, if system (1) has two equilibria $x_{1}^{*}, x_{2}^{*}$, then the general controller can be taken as the following simple form:

$$
\begin{aligned}
u\left(x, x_{1}^{*}, x_{2}^{*}, \mu\right)= & \left(x-x_{1}^{*}\right)\left(x-x_{2}^{*}\right)+\left(x-x_{1}^{*}\right)^{2}\left(x-x_{2}^{*}\right) \\
& +\left(x-x_{1}^{*}\right)\left(x-x_{2}^{*}\right)^{2} .
\end{aligned}
$$

If system (1) has only one equilibrium $x^{*}$, the controller can be taken as

$$
u\left(x, x^{*}, \mu\right)=\left(x-x^{*}\right)^{2}+\left(x-x^{*}\right)^{3} .
$$

We also omit the linear term $\left(x-x^{*}\right)$ in (6), because parameter $\alpha$ has the same control effect. In fact, it even can be more simple as

$$
u\left(x, x^{*}, \mu\right)=\left(x-x^{*}\right)^{3}
$$

For Hopf bifurcation control, as a result of the calculation formula of stability index [25], terms up to the third-order term are enough and the second-order term $\left(x-x^{*}\right)^{2}$ might not be necessary due to the presence of the third-order term $\left(x-x^{*}\right)^{3}$ for the simplicity of the calculation. The following are the conditions of system (2) undergoing Hopf bifurcation at the equilibrium.

Let $J(\mu)$ be the Jacobian matrix of system (2) evaluated at $x^{*}$. By the Hopf theory [25], $J(\mu)$ contains a complex conjugate pair of eigenvalues $\lambda_{1,2}(\mu)=\varphi(\mu) \pm \omega(\mu)$ satisfying

$$
\varphi(\tilde{\mu})=0, \quad \frac{d \varphi(\tilde{\mu})}{d \mu} \neq 0
$$

and the remaining eigenvalues of $J(\mu)$ have negative real parts at the critical point $\mu=\tilde{\mu}$. That is to say, when $\mu$ is varied, the pair of the complex conjugates moves to cross the imaginary axis at $\mu=\tilde{\mu}$. The second condition of (8) is usually called the transversality condition, implying that the crossing of the complex conjugate pair at the imaginary axis is not tangent to the imaginary axis. Without loss of generality, assume that when $\mu$ is varied from $\mu<\tilde{\mu}$ to $\mu>\tilde{\mu}, \lambda_{1,2}(\mu)$ moves from the left-half of complex plane to the right. Thus, a family of limit cycles will bifurcate from the equilibrium $x^{*}$ at the critical point $\tilde{\mu}$.

Next, it will be shown that the parameter $\alpha$ can change the bifurcation critical value, and the nonlinear state feedback can ensure the stability of bifurcation solutions.

\section{Hybrid Control of Hopf Bifurcation}

In this paper, the hybrid control is applied to Hopf bifurcation control of a new modified $4 \mathrm{D}$ hyperchaotic Lü system of the form [24]

$$
\begin{aligned}
& \dot{x}_{1}=a\left(x_{2}-x_{1}+x_{2} x_{3}\right), \\
& \dot{x}_{2}=-x_{1} x_{3}+b x_{2}+x_{4}, \\
& \dot{x}_{3}=x_{1} x_{2}-c x_{3}, \\
& \dot{x}_{4}=-k x_{1},
\end{aligned}
$$

where $x_{1}, x_{2}, x_{3}, x_{4}$ are state variables and $a, b, c, k$ are real parameters. System (9) has a hyperchaotic attractor when $a=35, b=14, c=3$, and $k=5$ [24]. Obviously, system (9) has only one isolated equilibrium $O(0,0,0,0)$ when $k \neq 0$. The controlled system is

$$
\begin{aligned}
& \dot{x}_{1}=\alpha_{1} a\left(x_{2}-x_{1}+x_{2} x_{3}\right)+\left(1-\alpha_{1}\right) x_{1}{ }^{3}, \\
& \dot{x}_{2}=\alpha_{2}\left(-x_{1} x_{3}+b x_{2}+x_{4}\right)+\left(1-\alpha_{2}\right) x_{2}{ }^{3}, \\
& \dot{x}_{3}=\alpha_{3}\left(x_{1} x_{2}-c x_{3}\right)+\left(1-\alpha_{3}\right) x_{3}{ }^{3}, \\
& \dot{x}_{4}=\alpha_{4}\left(-k x_{1}\right)+\left(1-\alpha_{4}\right) x_{4}{ }^{3} .
\end{aligned}
$$

$O(0,0,0,0)$ is also the equilibrium of system (10). The Jacobian matrix of system $(10)$ at $O(0,0,0,0)$ is

$$
A=\left(\begin{array}{cccc}
-a \alpha_{1} & a \alpha_{1} & 0 & 0 \\
0 & b \alpha_{2} & 0 & \alpha_{2} \\
0 & 0 & -c \alpha_{3} & 0 \\
-k \alpha_{4} & 0 & 0 & 0
\end{array}\right)
$$

The characteristic equation of $A$ is

$$
\begin{aligned}
\lambda^{4} & +\left(a \alpha_{1}-b \alpha_{2}+c \alpha_{3}\right) \lambda^{3} \\
& +\left(-a b \alpha_{1} \alpha_{2}+a c \alpha_{1} \alpha_{3}-b c \alpha_{2} \alpha_{3}\right) \lambda^{2} \\
& +\left(-a b c \alpha_{1} \alpha_{2} \alpha_{3}+a k \alpha_{1} \alpha_{2} \alpha_{4}\right) \lambda+a c k \alpha_{1} \alpha_{2} \alpha_{3} \alpha_{4}=0 .
\end{aligned}
$$


Taking $k$ as the Hopf bifurcation parameter and supposing that (12) has a pair of pure imaginary roots $\lambda_{1,2}= \pm i \omega_{0}\left(\omega_{0}>\right.$ $0)$, which leads to

$$
\begin{aligned}
& k_{0}=\frac{\alpha_{2} b^{2}-\alpha_{1} a b}{\alpha_{4}}, \\
& \omega_{0}=\sqrt{-a b \alpha_{1} \alpha_{2}} .
\end{aligned}
$$

The other two roots are

$$
\lambda_{3}=\alpha_{2} b-\alpha_{1} a, \quad \lambda_{4}=-c \alpha_{3} .
$$

Thus, the necessary conditions for system (10) to exhibit Hopf bifurcation at $O(0,0,0,0)$ are $a \alpha_{1}>0, b \alpha_{2}<0, c \alpha_{3}>0$, and $k=k_{0}$. Under these conditions, the transversality condition

$$
\begin{aligned}
& \operatorname{Re}\left(\left.\lambda^{\prime}\left(k_{0}\right)\right|_{\lambda=i \omega_{0}}\right) \\
& =\left(a \alpha _ { 1 } \alpha _ { 2 } \left[a \alpha_{1}\left(b \alpha_{2}-c \alpha_{3}\left(\alpha_{4}-1\right)\right)\right.\right. \\
& \left.\left.\quad-c \alpha_{3}\left(c \alpha_{3} \alpha_{4}-b \alpha_{2}\left(\alpha_{4}-1\right)\right)\right]\right) \\
& \quad \cdot\left(2\left(a^{2} \alpha_{1}^{2}-3 a b \alpha_{1} \alpha_{2}+b^{2} \alpha_{2}^{2}\right)\left(a b \alpha_{1} \alpha_{2}-c^{2} \alpha_{3}^{2}\right)\right)^{-1} \\
& \neq 0
\end{aligned}
$$

is also satisfied. Therefore, system (10) undergoes Hopf bifurcation at the equilibrium $\mathrm{O}(0,0,0,0)$ based on Hopf bifurcation theory [25].

Remark 1. In particular, if $\alpha_{1}=\alpha_{2}=\alpha_{3}=\alpha_{4}=1$, system (10) is reverted to the original system (9). The Hopf bifurcation value of the original system is $\widetilde{k}_{0}=b^{2}-a b$. So, parameter $\alpha$ can change the Hopf bifurcation value.

Remark 2. By formula (13), we notice that $\alpha_{3}$ does not affect the bifurcation critical value, so we can set $\alpha_{3}=1$ in system (10). And for simplicity, we also set $\alpha_{4}=1$ in the following discussion; that is to say, we may only choose the first two equations of system (9) under control. In this case, $k_{0}=\alpha_{2} b^{2}-$ $\alpha_{1} a b, \omega_{0}=\sqrt{-a b \alpha_{1} \alpha_{2}}$ are obtained.

\section{Analysis of Stability of Hopf Bifurcation}

In this section, we apply the normal form theory [25] to study the stability of the Hopf bifurcation for system (10).

By the linear transform $\left(x_{1}, x_{2}, x_{3}, x_{4}\right)^{T}=P\left(y_{1}, y_{2}, y_{3}\right.$, $\left.y_{4}\right)^{T}$, where

$$
P=\left(\begin{array}{cccc}
\frac{\omega_{0}}{b\left(\alpha_{1} a-\alpha_{2} b\right)} & 0 & -\frac{1}{b} & 0 \\
-\frac{\omega_{0} \alpha_{2}}{\alpha_{2}^{2} b^{2}+\omega_{0}^{2}} & -\frac{\alpha_{2}^{2} b}{\alpha_{2}^{2} b^{2}+\omega_{0}^{2}} & -\frac{\alpha_{2}}{\alpha_{1} a} & 0 \\
0 & 0 & 0 & 1 \\
0 & 1 & 1 & 0
\end{array}\right),
$$

then system (10) has the following normal form:

$$
\begin{aligned}
& \dot{y}_{1}=-\omega_{0} y_{2}+F_{1}\left(y_{1}, y_{2}, y_{3}, y_{4}\right), \\
& \dot{y}_{2}=\omega_{0} y_{1}+F_{2}\left(y_{1}, y_{2}, y_{3}, y_{4}\right), \\
& \dot{y}_{3}=\left(\alpha_{2} b-\alpha_{1} a\right) y_{3}+F_{3}\left(y_{1}, y_{2}, y_{3}, y_{4}\right), \\
& \dot{y}_{4}=-c y_{4}+F_{4}\left(y_{1}, y_{2}, y_{3}, y_{4}\right),
\end{aligned}
$$

where

$$
\begin{gathered}
F_{1}=-\frac{\alpha_{2}^{2}\left(a^{2} b \alpha_{1}^{2} \alpha_{2}\left(2 a \alpha_{1}-b \alpha_{2}\right) k_{1}+a^{3} \alpha_{1}^{3}\left(a \alpha_{1}-b \alpha_{2}\right) k_{2}\right)}{\omega_{0}^{5} k_{3}}, \\
F_{2}=\frac{a \alpha_{1}\left(k_{1}+k_{2}\right)}{b^{2} k_{3}}, \quad F_{3}=-F_{2}, \quad F_{4}=0, \\
k_{1}=\left(\alpha_{1}-1\right)\left(\alpha_{1} a y_{3}-\alpha_{2} b y_{3}-y_{1} \omega_{0}\right)^{3}, \\
k_{2}=\frac{\left(\alpha_{2}-1\right)\left(\alpha_{2}^{2} b^{2} y_{3}+a b \alpha_{1} \alpha_{2}\left(y_{2}-y_{3}\right)+\alpha_{1} a y_{1} \omega_{0}\right)^{3}}{a^{3} \alpha_{1}^{3}} \\
k_{3}=\left(\alpha_{1} a-\alpha_{2} b\right)^{2}\left(\alpha_{1}^{2} a^{2}-3 a b \alpha_{1} \alpha_{2}+\alpha_{2}^{2} b^{2}\right) .
\end{gathered}
$$

Then the stability condition of the bifurcated limit circle can be derived [25]:

$$
\begin{gathered}
\beta_{2}=\left(3 a \alpha _ { 1 } \alpha _ { 2 } \left(\alpha_{1}^{2} a^{2}\left(\alpha_{2}-1\right)+\alpha_{1} \alpha_{2} a b\left(5-2 \alpha_{1}-3 \alpha_{2}\right)\right.\right. \\
\left.\left.+\alpha_{2}^{2} b^{2}\left(-3+\alpha_{1}+2 \alpha_{2}\right)\right)\right) \\
\cdot\left(4 b\left(\alpha_{1} a-\alpha_{2} b\right)^{2}\left(\alpha_{1}^{2} a^{2}-3 \alpha_{1} \alpha_{2} a b+\alpha_{2}^{2} b^{2}\right)\right)^{-1} .
\end{gathered}
$$

If $\beta_{2}<0$, the bifurcated periodic solution is orbitally asymptotically stable, and if $\beta_{2}>0$, it is unstable. The following three special cases are considered.

Case 1. If $\alpha_{1}=\alpha_{2}=\alpha$, then $\beta_{2}=3 a\left(a^{2}-5 a b+3 b^{2}\right)(\alpha-$ 1) $/ 4 b(a-b)^{2}\left(a^{2}-3 a b+b^{2}\right)$. For $a>0, b<0$, we choose $\alpha>$ 1 ; then the bifurcation critical value of system (10) satisfies $k_{0}=\alpha\left(b^{2}-a b\right)>\widetilde{k}_{0}=b^{2}-a b$. Moreover, we have $\beta_{2}<0$.

Case 2. If $\alpha_{2}=1$, that is to say, only the first equation of system (9) is under control, then $\beta_{2}=-3 a \alpha_{1}\left(\alpha_{1}-1\right)\left(2 a \alpha_{1}-\right.$ b) $/ 4\left(b-a \alpha_{1}\right)^{2}\left(b^{2}-3 a b \alpha_{1}+a^{2} \alpha_{1}^{2}\right)$. For $a>0, b<0$, we choose $\alpha_{1}>1$; then the bifurcation critical value of system (10) satisfies $k_{0}=b^{2}-\alpha_{1} a b>\widetilde{k}_{0}=b^{2}-a b$. Moreover, we have $\beta_{2}<0$.

Case 3. If $\alpha_{1}=1$, that is to say, only the second equation of system (9) is under control, then $\beta_{2}=3 a \alpha_{2}\left(\alpha_{2}-1\right)(a-$ $\left.2 b \alpha_{2}\right) / 4 b\left(a^{3}-4 a^{2} b \alpha_{2}+4 a b^{2} \alpha_{2}^{2}-b^{3} \alpha_{2}^{3}\right)$. For $a>0, b<0$, we choose $\alpha_{2}>1$; then the bifurcation critical value of system (10) satisfies $k_{0}=\alpha_{2} b^{2}-a b>\widetilde{k}_{0}=b^{2}-a b$. Moreover, we have $\beta_{2}<0$. 


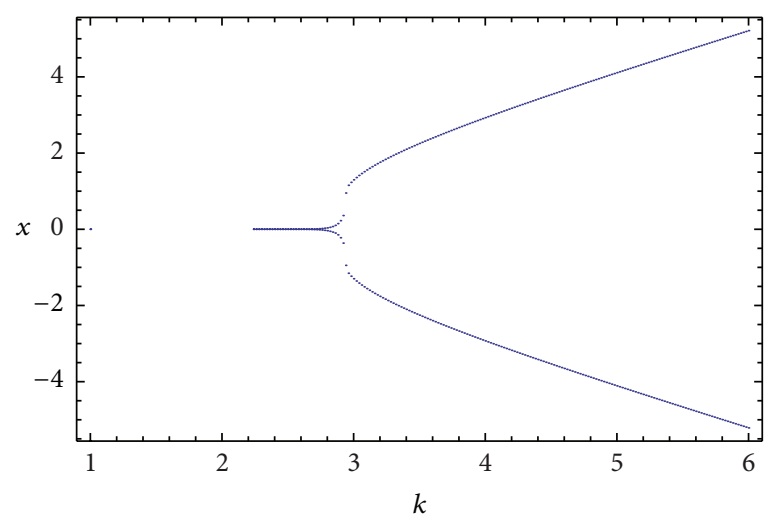

FIGURE 1: Bifurcation figure of original system with $a=2, b=-1$, and $c=2$.

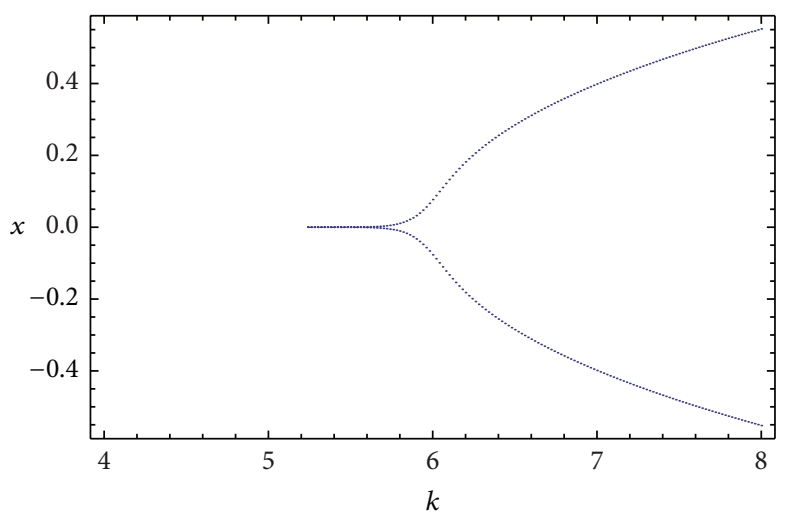

FIGURE 2: Bifurcation figure of controlled system with $a=2, b=-1, c=2$, and $\alpha_{1}=\alpha_{2}=2$.

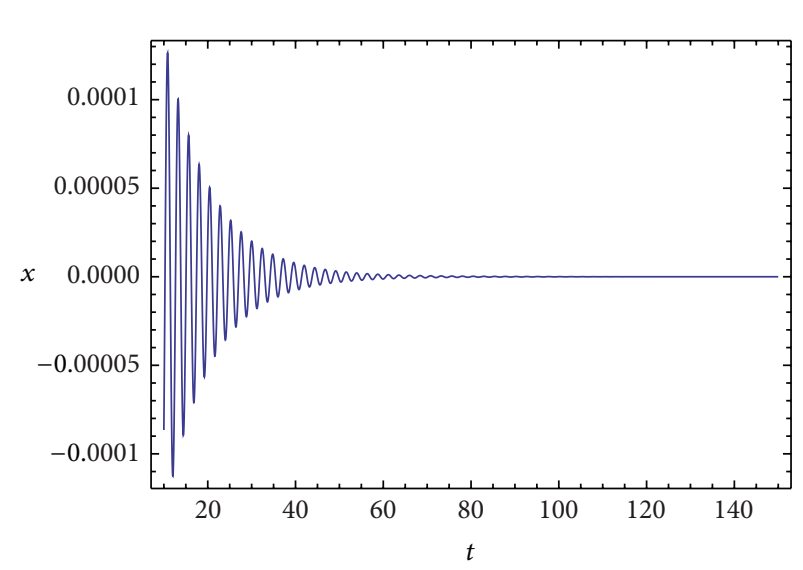

(a)

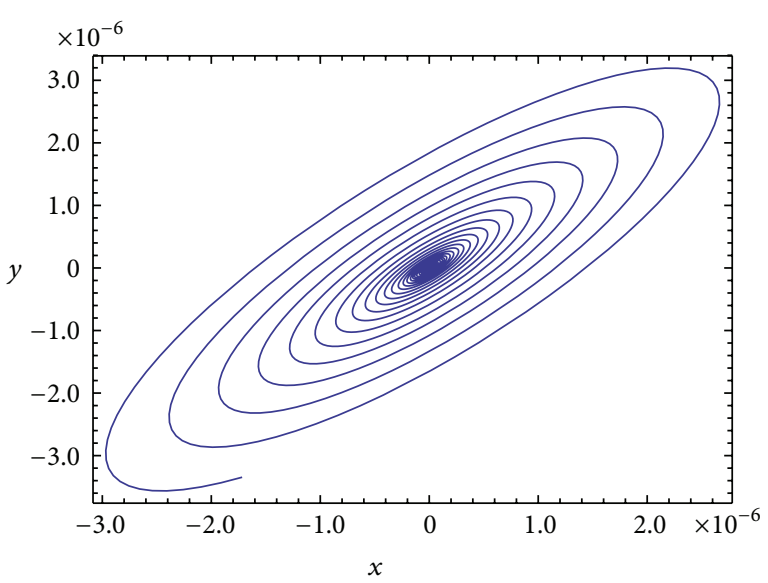

(b)

FIgure 3: Time displacement curves and phase space trajectories with $a=2, b=-1, c=2, \alpha_{1}=\alpha_{2}=2$, and $k=5$; (a) time displacement curves and (b) phase space.

So, theoretical analyses show that the control strategy not only delays Hopf bifurcation but also achieves the stability control of the bifurcation.

\section{Numerical Simulations}

In this section, numerical simulations are given to illustrate the above theoretical analyses. We choose $a=2, b=-1$, and $c=2$, and the original system (9) undergoes Hopf bifurcation at $\widetilde{k}_{0}=3$. The bifurcation figure of the original system (9) is shown in Figure 1. If we set $\alpha_{1}=\alpha_{2}=2$, the Hopf bifurcation critical value of the controlled system (10) is $k_{0}=6$, and $\beta_{2}=-0.257576$. The bifurcation figure of the controlled system (10) is shown in Figure 2. Time displacement curves and phase space trajectories are shown in Figures 3 and 4, respectively. 


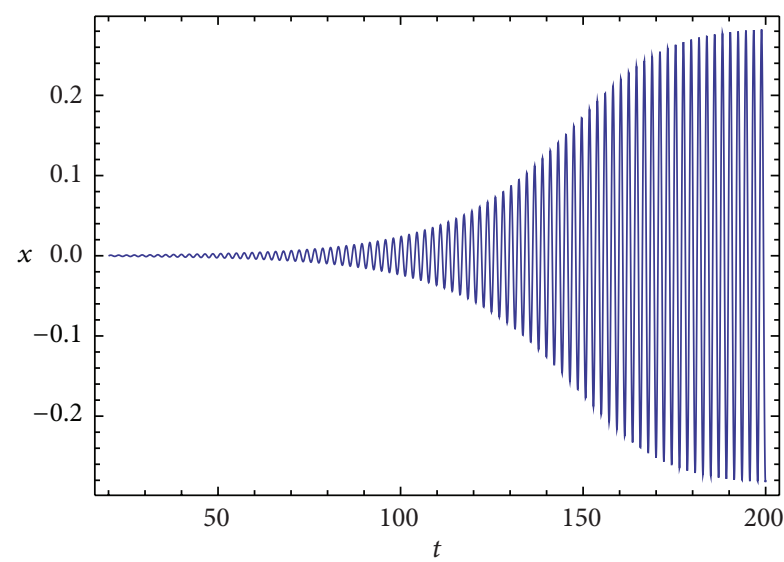

(a)

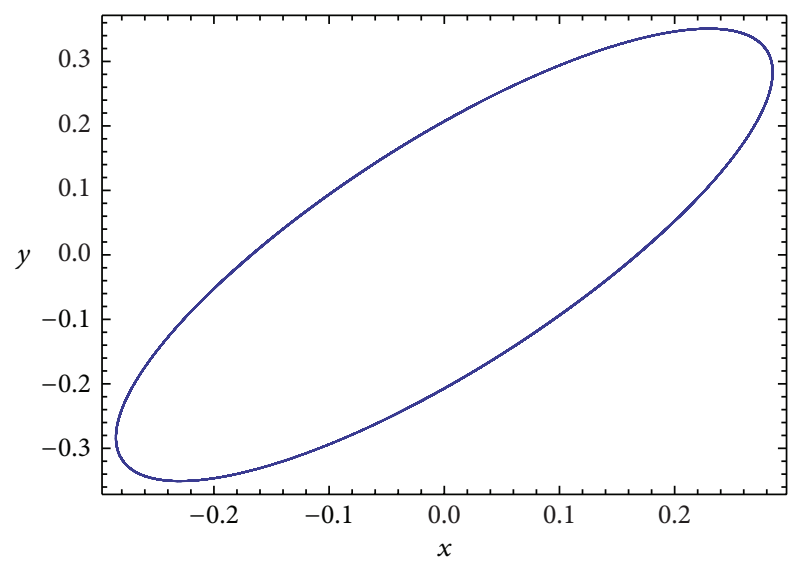

(b)

FIGURE 4: Time displacement curves and phase space trajectories with $a=2, b=-1, c=2, \alpha_{1}=\alpha_{2}=2$, and $k=6.5$; (a) time displacement curves and (b) phase space.

Therefore, the numerical simulation results are consistent with the theory analyses.

\section{Conclusions}

In this paper, a hybrid control strategy is applied to control the Hopf bifurcation in a new modified hyperchaotic Lü system for the first time. This method keeps the equilibrium construction of the original system and does not increase the dimension of the system. By choosing an appropriate control parameter, the control strategy can effectively delay the Hopf bifurcation, so the stable range of the system is extended. By using the normal form theory, the stability of bifurcating solutions is analyzed. Numerical simulations show the effectiveness of the method. Bifurcation control of high dimensional nonlinear systems is much more difficult than low dimensional systems. This control strategy is simple and convenient, so it is meaningful for the study of bifurcation control of high dimensional nonlinear systems.

\section{Conflict of Interests}

The authors declare that there is no conflict of interests regarding the publication of this paper.

\section{Acknowledgment}

The work is supported by the National Natural Science Foundation of China (11172093 and 11372102).

\section{References}

[1] G. M. Mahmoud and S. A. Aly, "Periodic attractors of complex damped non-linear systems," International Journal of NonLinear Mechanics, vol. 35, no. 2, pp. 309-323, 2000.

[2] W. Li, W. Xu, J. Zhao, and H. Wu, "The study on stationary solution of a stochastically complex dynamical system," Physica A, vol. 385, no. 2, pp. 465-472, 2007.
[3] G. M. Mahmoud, A. A. Mohamed, and S. A. Aly, "Strange attractors and chaos control in periodically forced complex Duffing's oscillators," Physica A: Statistical Mechanics and its Applications, vol. 292, no. 1-4, pp. 193-206, 2001.

[4] J. Lu, X. Yu, and G. Chen, "Chaos synchronization of general complex dynamical networks," Physica A: Statistical Mechanics and Its Applications, vol. 334, no. 1-2, pp. 281-302, 2004.

[5] G.-R. Jiang, B.-G. Xu, and Q.-G. Yang, "Bifurcation control and chaos in a linear impulsive system," Chinese Physics B, vol. 18, no. 12, pp. 5235-5241, 2009.

[6] X. D. Wang and L. X. Tian, "Bifurcation analysis and linear control of the Newton-Leipnik system," Chaos, Solitons and Fractals, vol. 27, no. 1, pp. 31-38, 2006.

[7] C.-X. Liang and J.-S. Tang, "Equilibrium points and bifurcation control of a chaotic system," Chinese Physics B, vol. 17, no. 1, pp. 135-139, 2008.

[8] J. S. Tang and Z. L. Chen, "Amplitude control of limit cycle in van der Pol system," International Journal of Bifurcation and Chaos, vol. 16, no. 2, pp. 487-495, 2006.

[9] J. Tang, F. Han, H. Xiao, and X. Wu, "Amplitude control of a limit cycle in a coupled van der Pol system," Nonlinear Analysis: Theory, Methods \& Applications, vol. 71, no. 7-8, pp. 2491-2496, 2009.

[10] L. H. Nguyen and K.-S. Hong, "Hopf bifurcation control via a dynamic state-feedback control," Physics Letters A, vol. 376, no. 4, pp. 442-446, 2012.

[11] P. Yu and G. Chen, "Hopf bifurcation control using nonlinear feedback with polynomial functions," International Journal of Bifurcation and Chaos in Applied Sciences and Engineering, vol. 14, no. 5, pp. 1683-1704, 2004.

[12] Z. Chen and P. Yu, "Hopf bifurcation control for an internet congestion model," International Journal of Bifurcation and Chaos in Applied Sciences and Engineering, vol. 15, no. 8, pp. 2643-2651, 2005.

[13] S. H. Liu and J. S. Tang, "Linear feedback control of the Hopf bifurcation in the Langford system," Acta Physica Sinica, vol. 56, no. 6, pp. 3145-3151, 2007 (Chinese).

[14] D. S. Chen, H. O. Wang, and G. Chen, "Anti-control of Hopf bifurcations," IEEE Transactions on Circuits and Systems. I. Fundamental Theory and Applications, vol. 48, no. 6, pp. 661672, 2001. 
[15] Z. Cheng, "Anti-control of Hopf bifurcation for Chen's system through washout filters," Neurocomputing, vol. 73, no. 16-18, pp. 3139-3146, 2010.

[16] Z. S. Lü and L. X. Duan, "Anti-control of Hopf bifurcation in the chaotic Liu system with symbolic computation," Chinese Physics Letters, vol. 26, no. 5, Article ID 050504, 2009.

[17] Z. C. Wei and Q. G. Yang, "Anti-control of Hopf bifurcation in the new chaotic system with two stable node-foci," Applied Mathematics and Computation, vol. 217, no. 1, pp. 422-429, 2010.

[18] H. W. Li and M. Wang, "Hopf bifurcation analysis in a Lorenztype system," Nonlinear Dynamics, vol. 71, no. 1-2, pp. 235-240, 2013.

[19] J. H. Lü, T. S. Zhou, G. R. Chen, and S. C. Zhang, "Local bifurcations of the Chen system," International Journal of Bifurcation and Chaos in Applied Sciences and Engineering, vol. 12, no. 10, pp. 2257-2270, 2002.

[20] Z.-S. Lü and L.-X. Duan, "Control of codimension-2 Bautin bifurcation in chaotic Lü system," Communications in Theoretical Physics, vol. 52, no. 4, pp. 631-636, 2009.

[21] S. H. Liu and J. S. Tang, "Anti-control of Hopf bifurcation at zero equilibrium of the 4D Qi system," Acta Physica Sinica, vol. 57, no. 10, pp. 6162-6168, 2008 (Chinese).

[22] C. X. Liang, J. S. Tang, S. H. Liu, and F. Han, "Hopf bifurcation control of a hyperchaotic circuit system," Communications in Theoretical Physics, vol. 52, no. 3, pp. 457-462, 2009.

[23] W.-G. Sun, Y. Chen, C.-P. Li, and J.-Q. Fang, "Synchronization and bifurcation analysis in coupled networks of discrete-time systems," Communications in Theoretical Physics, vol. 48, no. 5, pp. 871-876, 2007.

[24] G. Wang, X. Zhang, Y. Zheng, and Y. Li, "A new modified hyperchaotic Lü system," Physica A, vol. 371, no. 2, pp. 260-272, 2006.

[25] B. D. Hassard, N. D. Kazarinoff, and Y. Wan, Theory and Applications of Hopf Bifurcation, Cambridge University Press, London, UK, 1981. 


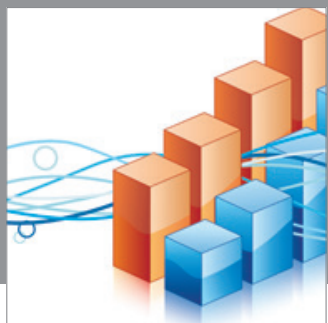

Advances in

Operations Research

mansans

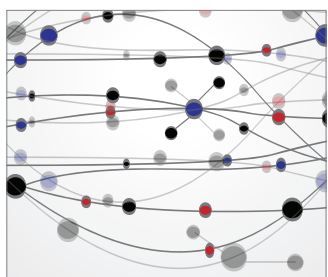

The Scientific World Journal
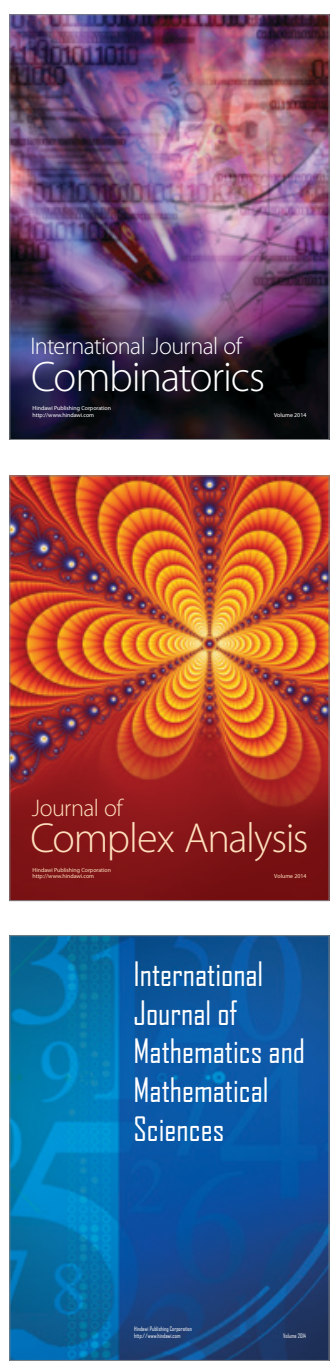
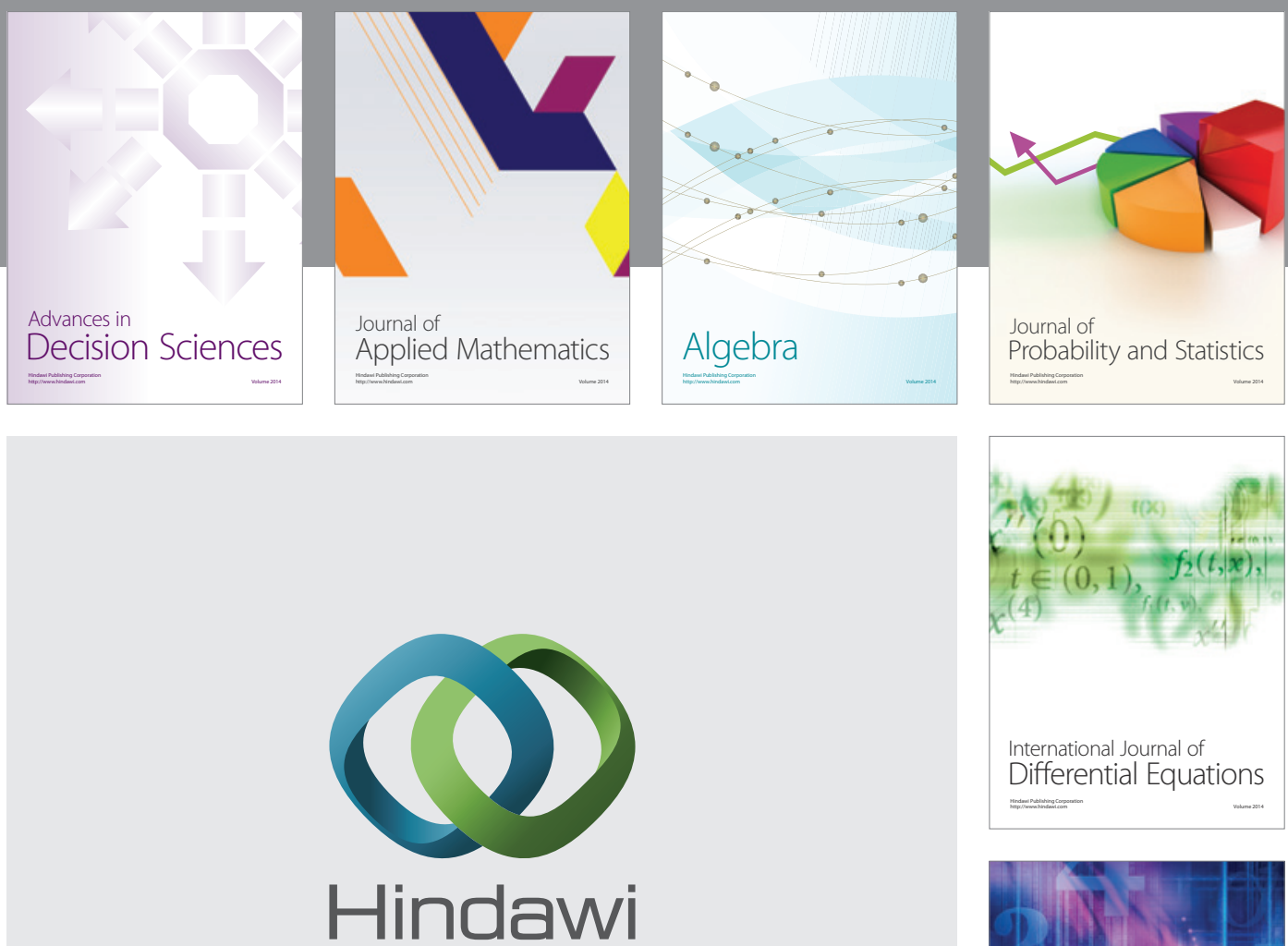

Submit your manuscripts at http://www.hindawi.com
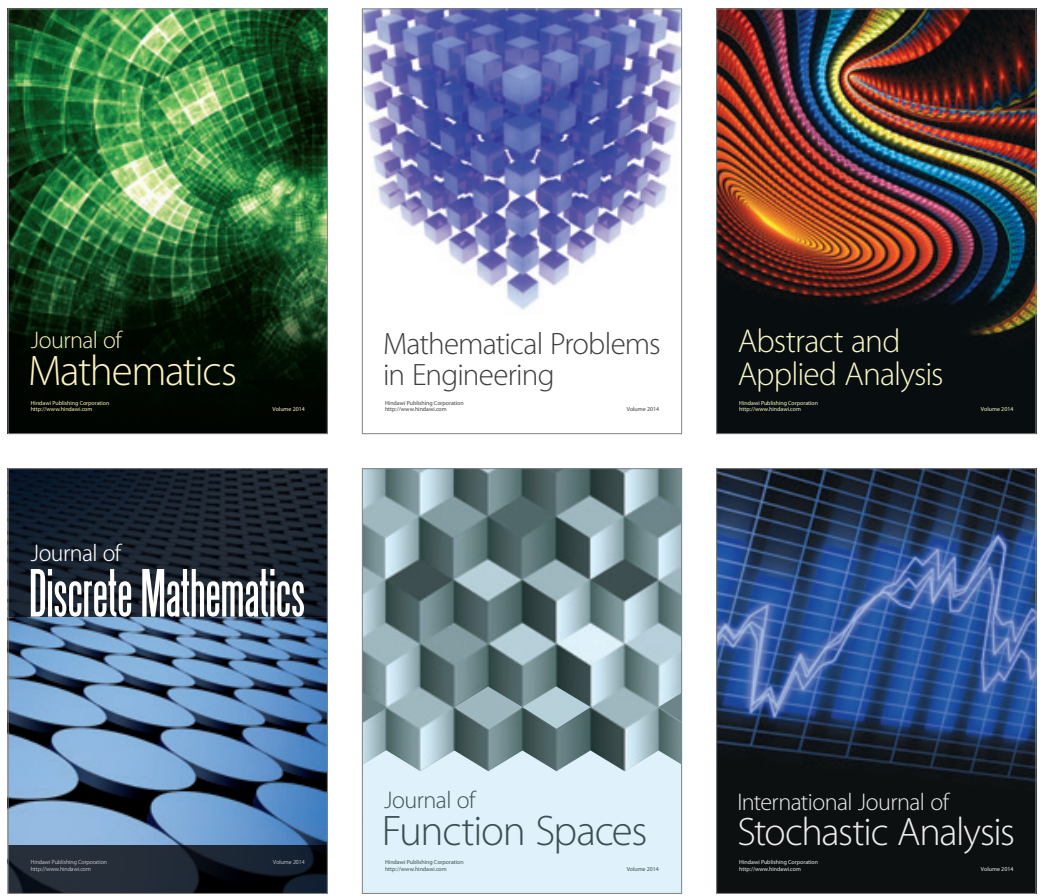

Journal of

Function Spaces

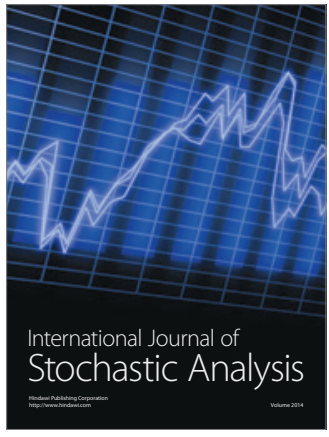

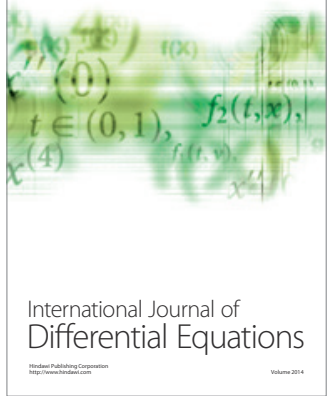
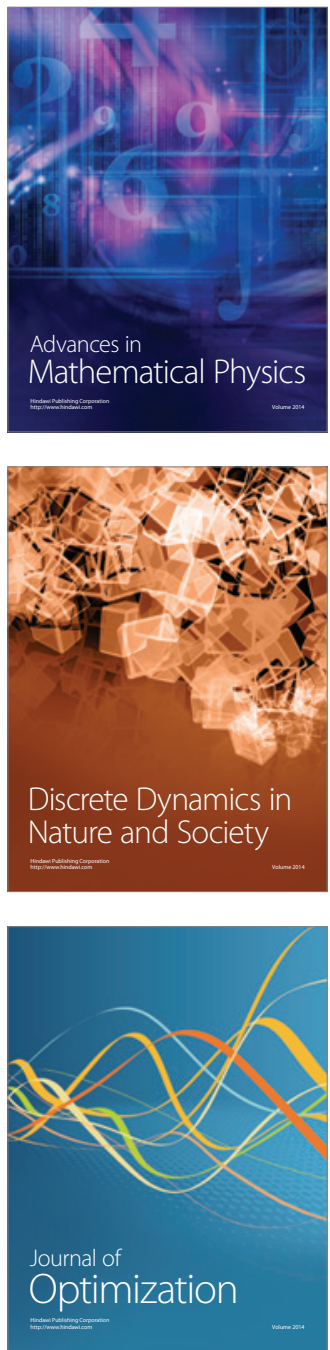